\title{
A novel, patient-specific mathematical pathology approach for assessment of surgical volume: Application to ductal carcinoma in situ of the breast
}

\author{
Mary E. Edgerton ${ }^{\mathrm{a}}$, Yao-Li Chuang ${ }^{\mathrm{b}, \mathrm{c}}$, Paul Macklin, ${ }^{\mathrm{c}, \mathrm{h}}$, Wei Yang ${ }^{\mathrm{d}}$, Elaine L. Bearer ${ }^{\mathrm{e}}$ \\ and Vittorio Cristini ${ }^{\mathrm{c}, \mathrm{f}, *}$ \\ ${ }^{a}$ Department of Pathology, UT MD Anderson Cancer Center, and School of Biomedical Informatics, \\ University of Texas Health Science Center, Houston, TX, USA \\ ${ }^{\mathrm{b}}$ Department of Pathology, Department of Mathematics, The University of New Mexico, Albuquerque, NM, USA \\ ${ }^{\mathrm{c} S c h o o l}$ of Biomedical Informatics, University of Texas Health Science Center, Houston, TX, USA \\ ${ }^{\mathrm{d} D e p a r t m e n t}$ of Diagnostic Radiology, UT MD Anderson Cancer Center, Houston, TX, USA \\ ${ }^{\mathrm{e}}$ Department of Pathology, The University of New Mexico, Albuquerque, NM, USA \\ ${ }^{\mathrm{f}}$ Department of Pathology, Department of Chemical Engineering, The University of New Mexico, Albuquerque, \\ NM, USA \\ ${ }^{\mathrm{g}}$ Division of Mathematics, University of Dundee, Dundee DD14HN, UK \\ ${ }^{\mathrm{h}}$ Center for Applied Molecular Medicine, Keck School of Medicine, University of Southern California, Los Angeles, \\ CA, USA
}

\begin{abstract}
We introduce a novel "mathematical pathology" approach, founded on a biophysical model, to identify robust patientspecific predictors of tumor growth useful in clinical practice to improve the accuracy of diagnosis/prognosis and intervention. In accordance with biological observations, our model simulates the diffusion-limited in-situ tumors with a relatively short phase of fast initial growth, followed by a prolonged slow-growth phase where tumor size is constrained primarily by the relative weight of cell mitosis and death. The former phase may only last for a few months, so that at the time of diagnosis, we may assume that most tumors will have entered the phase where their size is changing slowly. Based on this prediction, we hypothesize that the volume of breast with ducts affected by in-situ tumors at the time of diagnosis will be closely approximated by a modelderived mathematical function based on the ratio of tumor cell proliferation-to-apoptosis indices and on the extent of diffusion of cell nutrients (diffusion penetration length), which can be measured from immunohistochemical and morphometric analysis of patient histopathology specimens without the need for multiple-time measurements. We tested this idea in a retrospective study of 17 patients by staining breast tumor specimens containing ductal carcinoma in situ for mitosis with Ki-67 and for apoptosis with cleaved caspase- 3 and counting cells positive for each marker. We also determined diffusion penetration by measuring the thickness of viable rims of tumor cells within ducts. Using the ensuing ratios, we applied the model to determine a predicted surgical volume or tumor size. We then corroborated our hypothesis by comparing the predicted size of each tumor based on our model with the actual size of the pathological specimen after tumor excision $\left(\mathcal{R}^{2}=0.74-0.88\right)$. In addition, for the 17 cases studied, both histological grade and mammography were not found to correlate with tumor size $\left(\mathcal{R}^{2}=0.08-0.47\right)$. We conclude that our mathematical pathology approach yields a high degree of accuracy in predicting the size of tumors based on the mitotic/apoptotic index and on diffusion penetration. By obtaining these ratios at the time of initial biopsy, pathologists can
\end{abstract}

*Corresponding author: Vittorio Cristini, Department of Pathology, Department of Chemical Engineering, The University of New Mexico, Albuquerque, NM 87131, USA. E-mail: vcristini@salud. unm.edu. 
employ our model to predict the size of the tumor and thereby inform surgeons how much tissue to remove (surgical volume). We discuss how results from the model have implications concerning the current debate on recommendations for screening mammography, while the model itself may contribute to better planning of breast conservation surgery.

Keywords: DCIS, mathematical model, patient histology, IHC analysis, cell proliferation, cell death

\section{Introduction}

While tumor growth is a multifactorial dynamic phenomenon that evolves over time (e.g., months to years), clinicians often need to make diagnoses and decisions on treatments from one-time measurements of a few tumor properties, such as radiographic size and histologic patterns. Recent advances in biophysical models of tumor growth provide mathematical tools that could be employed to better predict tumor size based on measurements made in histological images of individual patients' tumors compared to the current approach, which is to use diagnostic imaging. We call this approach "mathematical pathology". Mathematical pathology is capable of distinguishing slow, long-term behaviors from fast, transient dynamics, thus identifying robust predictors of tumor growth that do not require measurements at multiple time points and may be readily incorporated within current clinical practice. In this paper, we apply a biophysical model of tumor growth [1] to predict tumor volumes of ductal carcinoma in situ with the long-term goal of employing this novel method to inform surgical planning for complete tumor excision.

Among the very few attempts to define new diagnostic/prognostic criteria using biophysical models, time-invariant "wavelengths" of roughness of the tumor boundary were identified as an important predictor of glioblastoma invasion through "fingering" into the brain stroma; the functional dependence of the fingering growth rates on tumor cell proliferation, apoptosis, and adhesion was calculated [2]. Furthermore, imaging data and mathematical models were used to predict glioma patient survival time and inform decisions on drug administration [3, 4]. Using mathematical models, it was also demonstrated that the growth of U87 glioblastoma converges to a constant rate, which can be directly linked to tumor proliferation, apoptosis, and vascularization [5].

There is a major unmet need for more accurate presurgical approaches to determine the size of a newly diagnosed breast cancer. Patients with DCIS (possibly the most prevalent precursor to invasive breast cancer
[6-11]) currently undergo some combination of breast conserving surgery, radiation therapy, and hormonal therapy. Breast conserving surgery fails to remove the entire tumor $38-72 \%$ of the time, requiring up to three surgeries for adequate tumor excision [12-14]. In the absence of adjuvant radiation and hormonal therapy, the estimated rate of recurrence after surgery is up to $25 \%$. With adjuvant radiation it is approximately $10 \%$ [15]. Half of these recurrences already show progression to invasive cancer, and at least $85 \%$ of the recurrences are either in the same site or in the same quadrant of the breast. The single most important underlying cause that contributes to both re-excisions and to recurrences has been attributed to malignant cells left inside the breast [16]. Mathematical pathology in combination with a breast imaging modality may be used to optimize surgical treatment of DCIS (in particular if accelerated partial breast irradiation is used [17]) by providing more accurate, patient-specific estimates of tumor volumes.

Mathematical analysis of the biophysics of diffusion-limited tumor growth in ductal carcinoma in situ of the breast leads to the hypothesis that insitu tumors with low cell mobility (e.g., non-pagetoid DCIS) begin with a relatively short phase of fast growth followed by a prolonged slow-growth phase. Thus DCIS tumors will progress to a slow growing, nearly stationary state where their final volumes depend mechanistically on the balance between tumor cell proliferation and tumor cell death. This balance depends on the diffusion of nutrients through the affected breast tissue and their penetration into the intraductal space. As we will demonstrate in this manuscript, the final volumes can be directly and quantitatively linked to the breast density, represented as the density of ducts within the breast tissue, and the relative rates of proliferation and apoptosis for the individual tumor.

\section{Materials and methods}

Previous mathematical and computational models of DCIS have focused on a single breast duct and 
have been successful in recapitulating certain features of the spatio-temporal dynamics of proliferating and motile tumor cells at this microscale [18-29], and have even, in some cases, been capable of extrapolating their results to predictions of macroscopic growth features [24] or invasive potentials as a function of grade [29]. Here we adopt a multiscale approach to predict growth and size of the volume of breast containing ducts with DCIS based on molecular measurements from histopathology of individual patient tumors. We thus aim not at predicting tumor volume within a single duct, but rather the gross volume of a breast lesion (herein denoted as surgical volume), which is currently the subject of mammographic imaging, and contains, among the various tissues: breast stroma, endothelium, fat, and ducts with DCIS within. Immunohistochemistry (i.e., Ki-67 and cleaved caspase-3 staining) and morphometric measurements (e.g., duct radius and thickness of viable rim of tumor cells within) are used to calibrate a cell-scale population dynamics model [24]. This information is then upscaled to a continuum, tissue-scale model [1] to estimate (surgical) tumor volumes. The present manuscript builds upon our preliminary work [30] with more patient data points, improved methods, and in-depth analysis of the results. This multiscale approach of mathematical pathology is illustrated in Fig. 1. We thus aim at providing a tool for more accurate assessment of surgical volume, which could improve the success of complete excision of breast tumors.

Table 1 lists the definitions of all the model parameters and variables used in this paper. The parameter input values calculated from raw pathologic measurements are summarized in Table 2 and described in the following.

\subsection{A mathematical formula for predicting the size of in-situ tumors}

We model the surgical volume as a porous medium (of roughly ellipsoidal shape) with tumor cells growing through it [1]. In this preliminary version of the model we are not interested in the detailed geometry of the duct system and other tissues within this volume of breast, but rather in accurately assessing the extent of the volume required for adequate surgical excision.

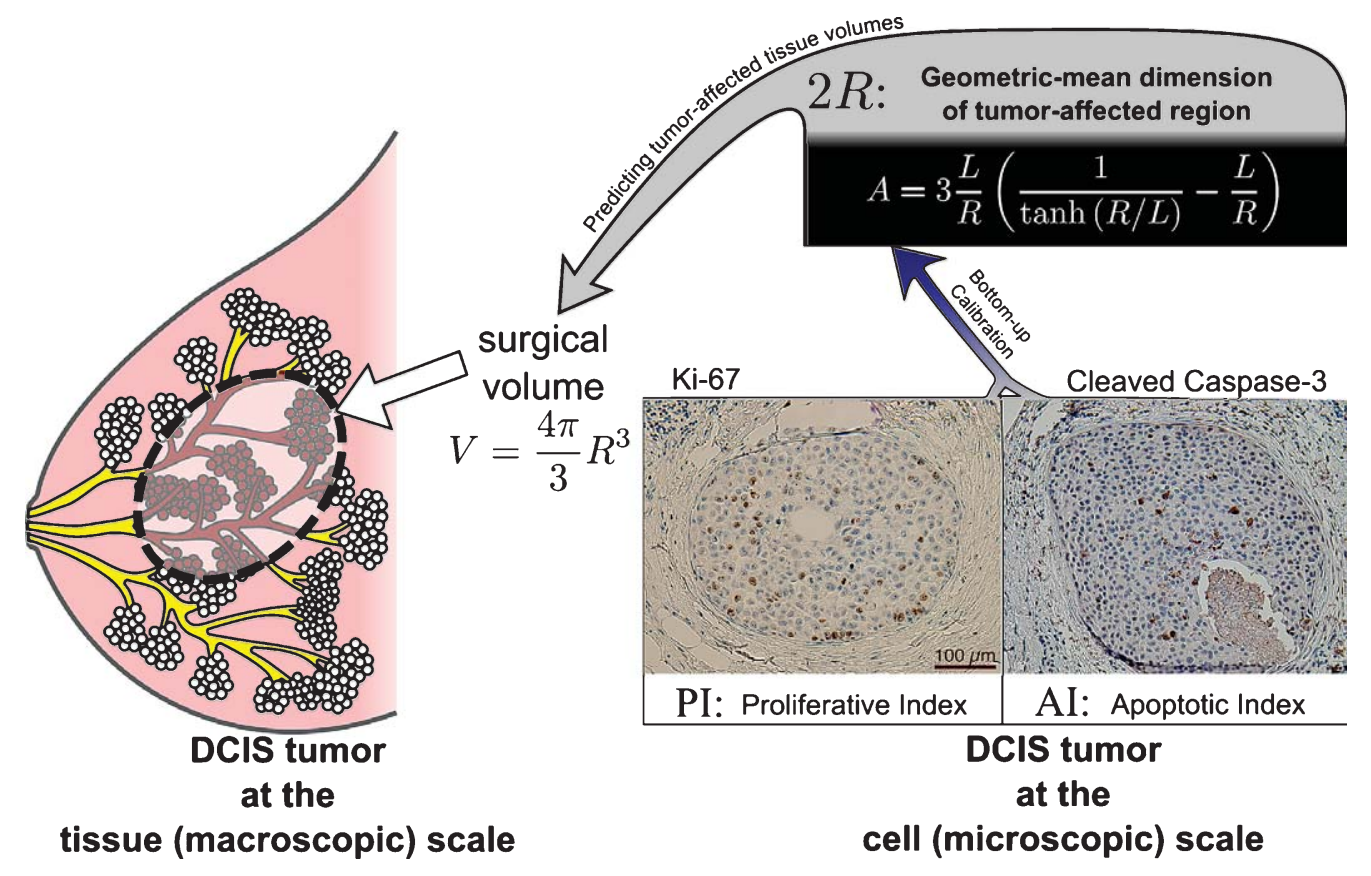

Fig. 1. Patient-specific model prediction of surgical volume from pathology data. The cell-scale measurements (e.g., proliferation and apoptotic indices) are averaged across each tumor to calibrate the tissue-scale model, which then predicts surgical volume [5]. The key formula (Eq. 1) of the model depends mechanistically on the pathology-measurable patient-specific parameters $L$ and $A$, i.e., nutrient diffusion-penetration length in the tumor and ratio of tumor cell death to proliferation. The left portion of the figure is adapted from [30, Fig. 10.1], reprinted with permission from Cambridge University Press. 
Table 1

Biophysical model parameters

\begin{tabular}{ll}
\hline Parameter & Biophysical meaning \\
\hline$A$ & Ratio of cell apoptosis to proliferation rates \\
& (Eqs. 1, 2a) \\
AI, PI & Apoptotic, proliferative indices (Eq. 3) \\
$D, \lambda$ & Nutrient diffusion coefficient and uptake rate by \\
& tumor cells (Eq. 2b, footnote 1) \\
$f$ & Tumor volume fraction (Eq. 2b, footnote 1) \\
$L$ & Nutrient diffusion penetration length across \\
$R$ & tumor surgical volume (Eqs. 1, 2b) \\
$R_{\mathrm{duct}}, T$ & Geometric-mean tumor surgical radius (Eq. 1) \\
$\lambda$ & Duct radius, viable-rim thickness (Eq. 4) \\
$\sigma, \sigma_{\mathrm{H}}$ & Tumor cell apoptosis, mitosis rates (Eqs. 2a, 3) \\
$\tau_{\mathrm{A}}, \tau_{\mathrm{P}}$ & Nutrient concentration, concentration at the \\
\hline & peri-necrotic boundary (Eq. 4, footnote 1)
\end{tabular}

Therefore we average local phenotypic and morphometric properties. In the Discussion, we present ideas for extending this analysis to accurate prediction of tumor shape (e.g., the ratios of the three dimensions as measured from mammography).

In this model, cell proliferation and death, together with nutrient diffusion and uptake by the cells, uniquely determine the rate of growth and the tumor size. Analysis [1] of the model reveals that the geometric mean of the tumor dimensions (i.e., the cube-root of the surgical volume or, as expressed in our model, the diameter $2 R$ ) reaches a (nearly) stationary value, which is set by an overall balance of mass gain from proliferation in well oxygenated areas and mass loss from cell death in hypoxic or nutrient-depleted areas. Our simulations using a range of physiological input values (as described below) show that DCIS tumors reach nearly stationary sizes following a short period of fast growth which may last as little as about two months [31, 32]. Given such short growth time compared to yearly screenings by mammogram, we may expect most DCIS tumors to be near their stationary size at the time of diagnosis. The value of $R$ depends upon two key parameters: $A$, which is the ratio of cell-death to cell-proliferation rate constants averaged over the multitude of ducts within the surgical volume, and $L$, the nutrient (e.g., oxygen) diffusion penetration length within the involved breast tissue, via the following mathematical pathology formula [1]:

$$
A=3 \cdot \frac{L}{R} \cdot\left(\frac{1}{\tanh (R / L)}-\frac{L}{R}\right)
$$

Table 2

Summary of pathological/mammographic features with model parameter values and predictions for index series*

\begin{tabular}{|c|c|c|c|c|c|c|c|}
\hline \multirow[t]{2}{*}{ Case ID } & \multirow[t]{2}{*}{ Subtype } & \multirow[t]{2}{*}{ Grade ${ }^{* * *}$} & \multirow[t]{2}{*}{$A$} & \multirow[t]{2}{*}{$L(\mu \mathrm{m})$} & \multicolumn{3}{|c|}{ Diameter $(\mathrm{cm})$} \\
\hline & & & & & Model $^{\mathrm{a}}$ & Image $^{b}$ & Path $^{\mathrm{c}}$ \\
\hline 8 & Cribriform & 2 & $2.00 \mathrm{E}-02$ & 374.00 & 11.14 & 1.56 & 0.93 \\
\hline 13 & Solid & 3 & 4.11E-02 & 196.84 & 2.83 & 2.22 & 3.27 \\
\hline 14 & Cribriform & 2 & $3.01 \mathrm{E}-03$ & 350.75 & 69.96 & 1.43 & 1.15 \\
\hline 15 & Cribriform & 1 & $1.15 \mathrm{E}-01$ & 301.63 & 1.51 & 1.14 & 0.96 \\
\hline 17 & Mixed $^{* *}$ & 2 & $2.17 \mathrm{E}-01$ & 222.33 & 0.57 & 0.93 & 1.13 \\
\hline 18.1 & Cribriform & 1 & 2.63E-01 & 237.50 & 0.49 & 0.79 & 1.06 \\
\hline 18.2 & Mixed $^{* *}$ & 3 & 4.66E-02 & 228.03 & 2.89 & 0.80 & 3.27 \\
\hline 19 & Mixed $^{* *}$ & 3 & $2.75 \mathrm{E}-02$ & 160.99 & 3.48 & 6.11 & 2.27 \\
\hline 21 & Cribriform & 2 & 5.79E-02 & 230.88 & 2.35 & 1.98 & 2.05 \\
\hline 22 & Cribriform & 3 & $3.92 \mathrm{E}-02$ & 198.16 & 3.00 & 4.64 & 2.08 \\
\hline 23 & Solid & 3 & $1.06 \mathrm{E}-01$ & 275.12 & 1.51 & 1.64 & 1.16 \\
\hline 28 & Solid & 3 & $3.90 \mathrm{E}-02$ & 176.73 & 2.69 & 1.13 & 2.93 \\
\hline 39 & Mixed $^{* *}$ & 1.5 & $3.83 \mathrm{E}-02$ & 158.30 & 2.45 & 1.29 & 1.5 \\
\hline 40 & Mixed** & 1.5 & $5.31 \mathrm{E}-02$ & 457.05 & 5.07 & 5.01 & 4.58 \\
\hline 42 & Cribriform & 1 & $4.90 \mathrm{E}-02$ & 303.53 & 3.65 & 5.00 & 3.44 \\
\hline 48 & Cribriform & 1 & $2.85 \mathrm{E}-02$ & 278.18 & 5.80 & 2.82 & 2.38 \\
\hline 51 & Solid & 3 & $9.08 \mathrm{E}-02$ & 218.23 & 1.40 & 3.02 & 1.39 \\
\hline
\end{tabular}

* Volume fraction $f=24 \%$ (average of all 17 cases) was used.

** Mixed Subtype denotes mixed solid and cribriform subtypes.

*** Grade of 1.5 is a numerical representation of a low/intermediate grade assigned by a pathologist.

a $2 R$ from Eq. (1).

${ }^{\mathrm{b}}$ Based on geometric mean of three dimensions measured from mammographic images.

${ }^{\mathrm{c}}$ Based on geometric mean of three dimensions measured from pathology material. 
Thus we are assuming that increased uptake due to the presence of the tumor cells in the DCIS-affected ducts leads to conditions of oxygen and nutrient depletion within the tumor surgical volume, and eventually to diffusion-limited growth of this volume. Note that this hypothesis only applies to non-pagetoid DCIS, where cell migration along the basement membrane can be neglected.

It would be problematic to input cell-scale (i.e., $<200 \mu \mathrm{m}$ ) patient-specific histological and immunohistochemical molecular measurements directly to whole-tumor models (e.g., at the centimeter scale) of surgical volume. To obtain the patient-specific values of $A$ and $L$, and thus predict tumor surgical volumes $4 \pi R^{3} / 3$, we introduce a multiscale approach, where immunohistochemistry and morphometric measurements of the individual ducts from patient resected tissue (see below) are used to directly calibrate key parameters in a cell-scale population model. These parameters are subsequently upscaled through biophysically founded "static" mathematical relations to accurately inform the parameters $A$ and $L$ of the tissue-scale continuum model Eq. (1). This mathematical pathology procedure is described in detail in the following. The definitions of the parameters are $[1,30]$ :

$$
A=\lambda_{\mathrm{A}} / \lambda_{\mathrm{M}} ; \quad L=f^{-1 / 2} \cdot(D / \lambda)^{1 / 2} ;
$$

where the maximum mass growth rate constant by mitosis in ducts is $\lambda_{\mathrm{M}}$ (i.e., corresponding to nutrient conditions of $\sigma=1$ ), and the analogous death (e.g., apoptosis) rate constant is $\lambda_{\mathrm{A}}$ (all calculated by their average values throughout the patient tumor surgical volume). The term $\sigma$ represents the local nutrient (e.g., oxygen) concentration normalized to the concentration in the uninvolved breast tissue away from the DCISaffected regions (i.e., $\sigma<1$ inside the breast tissue involved by DCIS and $\sigma=1$ outside the region of tumor involvement). The (average) nutrient diffusion coefficient in ducts is $D, f$ is the fraction of surgical volume occupied by viable tumor cells and $\lambda$ is the nutrient uptake rate by cells in ducts. Thus the quantity $L$ is an average property of breast tissues within the surgical volume that characterize how far oxygen and nutrients may propagate by diffusion under uptake by the cells (including the DCIS cells) [1] (the quantity $(D / \lambda)^{1 / 2}$ is the corresponding average intraductal diffusion penetration length, reflecting the fact that the cells are placed within a heterogeneous distribution of oxygen and nutrients diffusing from the vessels outside the duct walls into the duct interior) ${ }^{1}$.

\subsection{Patient-specific calibration of Eqs. (1) and (2) from cell-scale measurements}

By volume averaging, we set the rates of mass growth (due to DCIS cell mitosis) and decrease (death) within the surgical volume by averaging over corresponding proliferation rates in a cell population model [24, 30, 33]:

$$
\lambda_{M} \cdot\langle\sigma\rangle=\mathrm{PI} / \tau_{\mathrm{p}} ; \text { and } \lambda_{A}=\mathrm{AI} / \tau_{\mathrm{A}} ;
$$

and thus determine $\lambda_{M}$ and $\lambda_{A}$. Here, PI and AI are the proliferative and apoptotic indices (fractions of total number of cells) respectively. We measured PI using $\mathrm{Ki}-67$ to identify cycling (non- $\mathrm{G}_{0}$ ) cells and AI using cleaved caspase- 3 to measure the fraction of cells in the apoptotic state (see below). Values are measured for individual ducts by IHC and averaged over the ducts of each tumor case (Table S1). In the population model ${ }^{2}$, $\tau_{\mathrm{P}}$ is the (constant) duration of the cell cycle; cell death processes (e.g., apoptosis) have time duration $\tau_{\mathrm{A}}$. We set $\tau_{\mathrm{P}}=18$ hours to complete a cell cycle and proliferate [37]. We set $\tau_{\mathrm{A}}=6.6$ hours [24, 30,33] by applying the population model to (benign) breast epithelium [38] and correcting for the early portion of apoptosis that cannot be detected by TUNEL assay but is detected by cleaved caspase-3 [39]; this estimate is consistent with the experimental literature (e.g., [40, 41]). The diffusion penetration length $L$ is calculated from Eq. (2b), where we measure $f$ as the fraction of area occupied

\footnotetext{
${ }^{1}$ Eqs. (1) and (2) can be derived [1] by solving corresponding conservation equations within the surgical volume: $\nabla \cdot \mathbf{u}=\lambda_{\mathrm{M}}$. $\sigma-\lambda_{\mathrm{A}}$, for the surgical volume growth rate, and $D \cdot \nabla^{2} \sigma-f \cdot \lambda$. $\sigma=0$, for nutrient transport within this volume, where $\mathbf{u}$ is the local cell velocity due to proliferation-induced volume expansion, and the latter describes diffusion and local rate of uptake of nutrients by (tumor) cells.

2 We apply herein a reduced version of a general agent-based cell model [24, 30, 33, 34] (preprint of [24] at: http://www. MathCancer.org/Publications.php\#macklin11_jtb) that improves over a previous cellular automaton approach $[35,36]$. Accordingly, we only determine averaged tumor population dynamics of mitosis and death, where these phenotypic states are governed by stochastic processes which depend upon the cells' internal machinery and its sampling of the heterogeneous microenvironment (nutrient concentrations $\sigma$ ). The population dynamics can be averaged across the viable rim within a duct to obtain an equation governing the total number $N$ of viable cells therein: $\mathrm{d} N / \mathrm{d} t=\left(\mathrm{PI} / \tau_{P}-\mathrm{AI} / \tau_{A}\right) \cdot N$, and then derive Eq. (3).
} 
by viable tumor (duct space occupied by viable tumor) from the histopathology images as described below.

The overall population dynamics is substratedependent. We estimate the mean nutrient level across the viable rim of tumor cells (within an idealized cylindrical duct of radius $R_{\text {duct }}$ and viable-rim thickness $T$ ) in Eq. (3a) by solving the diffusion equation (see footnote 1) to obtain [24, 30]:

$$
\begin{aligned}
\langle\sigma\rangle= & \frac{\sigma_{H}}{I_{0}\left(\frac{R_{\text {duct }}-T}{L_{0}}\right)} \cdot \frac{2 L_{0}}{2 R_{\text {duct }} T-T^{2}} \\
& \cdot\left[R_{\text {duct }} \cdot I_{1}\left(\frac{R_{\text {duct }}}{L_{0}}\right)-\left(R_{\text {duct }}-T\right)\right. \\
& \left.\cdot I_{1}\left(\frac{R_{\text {duct }}-T}{L_{0}}\right)\right],
\end{aligned}
$$

where $\sigma_{\mathrm{H}}$ is the threshold concentration value at a distance $T$ from the duct wall (viable-rim thickness), below which cells become necrotic due to lack of oxygen and nutrients (we set $\sigma_{H}=0.2[24,30]$ based upon published models for breast cancer [37, 42]), and $I_{n}$ is the $n$-th order modified Bessel function of the first kind. We use the average duct radius $\left\langle R_{\text {duct }}\right\rangle$ and viable-rim thickness $\langle T\rangle$ values from duct morphometric measurements (see below) in place of $R_{\text {duct }}$ and $T$ in Eq. (4). We set [30] the intraductal diffusion length $L_{0}=(D / \lambda)^{1 / 2}$ by averaging the measurements of $T$ in several ducts in resected tissue for each patient: $L_{0}=<T>$ (Table S2). Note that this measurement requires necrosis to be present in the histological sections of DCIS.

\subsection{Selection of cases and immunohistochemistry}

Our index series of 17 cases has DCIS tumors ranging in maximum dimension from $1-9.8 \mathrm{~cm}$ with a median value of $4.0 \mathrm{~cm}$. Among these 17 cases, 12 of them, also used in our preliminary study [30], were collected and processed in 2008 (Cases 8-28), while the other five were obtained in 2009 (Cases 39-51). Our samples include four solid-type $(23.5 \%)$, six cribriform $(35.3 \%)$, and seven solid / cribriform mixed-type $(41.2 \%)$ growth patterns. Grade distribution for our series was as follows: four cases $(23.5 \%)$ were low, two $(11.8 \%)$ were low/intermediate (represented by a numerical grade of 1.5 in Table 2 ), four $(23.5 \%)$ were intermediate, and seven (41.2\%) were high grade. A minimum of one and maximum of three formalin fixed paraffin embedded (FFPE) blocks of tumor tissue were selected from each case for immunohistochemistry measurements. Blocks were selected that contained the highest density of tumor cells. Each block had a minimum of $1 \mathrm{~cm}^{2}$ surface area of breast tissue for examination. Five (5) micron sections were cut for staining either with Hematoxylin and Eosin (H\&E) to visualize the tumor, or with immunohistochemistry for a specific antibody to identify and quantify a particular antigen and its subcellular location at a minimum resolution of approximately $2 \mu \mathrm{m}$ (spatial resolution equivalent to quarter-width of nucleus). We measured the proliferative index (PI) as the fraction of cells that were Ki-67 positive. $\mathrm{Ki}-67$ is a nuclear antigen that is expressed throughout cell cycle, except during portions of the $G_{1}$ phase, and is the gold standard for measuring PI (e.g., [43]). Ki-67 was stained with MIB-1 (clone of antibodies from Dako). Cleaved caspase-3, an executioner protein that has been documented as an apoptotic marker throughout most of the apoptotic cycle (e.g., [44]), was used to quantify the apoptotic index AI, i.e., the fraction of cells in the apoptotic state. Cleaved caspase- 3 was stained using antibody purchased from Biocare Medical. For both stains, five micron sections were hydrated to water and antigen retrieved in citrate buffer $\mathrm{pH} 6$. The sections were blocked with $3 \%$ hydrogen peroxide for 5 minutes, whole goat serum for 5 minutes, incubated with antibody to either $\mathrm{Ki}-67$ or cleaved caspase-3 for 30 minutes followed by goat anti-rabbit IgG-horse radish peroxidase (HRP) for 15 minutes, and then localized with diaminobenzidine (DAB) for 5 minutes. Slides were counterstained with Mayer's hematoxylin for 5 minutes, dehydrated and cover-slipped.

Quantification for AI and PI on immunohistochemistry stained sections was performed as follows. Magnified images $(100 \times$ and $200 \times)$ of multiple areas of DCIS were analyzed using computational image processing routines. The image samples of the 2008 batch were preprocessed by a custom-built Visual Basic color-thresholding plug-in for Image Pro Plus 4.5 to quantify the total number of tumor cells (denominator to $\mathrm{AI}$ and $\mathrm{PI}$ ) and the number of $\mathrm{Ki}-67$ positive nuclei (numerator for PI) in a selected duct. The image samples of the 2009 batch were preprocessed and counted by a MATLAB program developed by our group, using three-cluster k-means to distinguish the positive and the negative nuclei and the image background $^{3}$ [45]. Images that were too faint for

\footnotetext{
${ }^{3}$ Preprint to be made available at http://www.MathCancer.org
} 
the computational routines to distinguish the stained cells from the background were counted manually. We counted a minimum of 1891 cells per tumor specimen (Table S1). We expect the k-means algorithm, which can achieve better separation of the chromogen stain color space versus the background stain color space on IHC because it is using the individual spectral output of the images, to be more consistent than grayscalethresholding techniques currently in widespread use, which do not use spectral information but rely on relative intensity of colors. Based on 21 randomly selected duct images, the individual results for PI and AI generated by the k-means algorithm differed from the manual cell counts by $23.2 \%$ on average. The resulting difference in the parameter $A$ values for the same images was only $6.4 \%$. We attributed the differences in cell counts for AI and PI to systematic errors due to image quality that partially canceled each other when $A$ was calculated form their ratio. Thus we conclude that the calculation of A is quite robust against systematic counting errors. We also note that the total number of cells is approximately the same in both the $\mathrm{Ki}-67$ and the cleaved caspase- 3 sections; thus AI/PI reduces to the ratio of positive cleaved caspase- 3 cells to positive Ki-67 cells. The automated and manual counts of the positive cells are more robust due to the darker color of the positive stain when grayscale thresholding is used, and to the spectral separation of the positive stain when the k-means algorithm is used. In addition, the smaller number of cells to count improves the manual accuracy.

The IHC counts for each case are given in Table $S 1$ in the Supplementary Data. We estimated the variations of $A$ (Eq. 3) using Taylor expansion of the standard errors of the mean (SEM) of the PI and AI measurements performed on individual duct cross sections for each tumor $[46,47]$, thus accounting for the combined effects of intra- tumor heterogeneity (e.g., due to phenotypic and microenvironmental differences), sample sizes and measurement errors.

There was significant variation between cases. Among the 17 cases, one intermediate grade cribriform type case (Case 21) did not display any positive staining for cleaved caspase-3. In addition, one highgrade case (Case 13) displayed a high background of non-specific cleaved caspase-3 staining suggesting that the staining procedure failed. Given the clustering of values for AI and PI that was observed as a function of grade (see discussion that follows) the values for AI for these two cases was substituted with the mean value for $\mathrm{AI}$ for the remaining intermediate and high grade tumors, respectively.

\subsection{Tumor-size and morphometric measurements}

Tumor size was estimated by reviewing the pathology gross description and individual tumor sections. The dimensions for small DCIS present on a single slide $(<1 \mathrm{~cm}$ in greatest extent) were estimated by measuring the span on a single slide using a measuring tool (Olympus BX 41 with ocular micrometer). For larger DCIS, the width was estimated by multiplying the number of slices containing DCIS by the average width of a slice. The standard procedure for DCIS surgical specimens is serially slicing the gross specimen from medial to lateral. The thickness of individual slices was estimated by dividing the width of the specimen by the total number of serial slices. Width in the medial to lateral plane was estimated using the number of slices involved multiplied by the average width of a slice. Adjacent sections that represented DCIS in its entirety in a cross section of either the anterior-posterior and superior-inferior planes were laid out and the extent of the DCIS estimated by measuring the distance between the furthest points to which it extended across the glass slides. This approach is similar to that used in the literature to estimate pathologic size of DCIS (e.g., [48]) and is considered the most accurate means of measuring tumor size [49]. The 17 cases used in this paper did not include any multicentric DCIS, which is considered to be independent tumors present in more than one quadrant [50].

Typically DCIS is characterized by the largest measurement along any single dimension, called the "greatest dimension" of the tumor. We used instead the "geometric mean" diameter (the cube-root of the product of the three measured dimensions of the tumor, which is a better indicator of the volume), and compared this to the diameter predicted by Eq. (1). Although the greatest dimension for our series of ductal carcinoma cases was as high as $9.8 \mathrm{~cm}$, none of the tumors had a geometric mean larger than $4.58 \mathrm{~cm}$. The full list of measured dimensions is reported in Table S3.

Mammographic measurements of tumor dimensions were performed by reviewing the images and determining the maximum distance between suspicious calcifications belonging to the target lesion in the sagittal (anterior-posterior), transverse (medial-lateral), and 
coronal (superior-inferior) planes. As for the pathology specimens, the geometric mean of the mammographic dimensions was calculated, while the full list of the measurements is reported in Table S3.

We measured $f$ (the volume fraction of the viable tumor) by taking multiple H\&E images of DCIS at $40 \times$ and calculating the fraction of area occupied by viable DCIS in the representative blocks selected for study. If adipose tissue was present in the slides, the corresponding area was not included in the denominator of $f$ for consistency. Our basis for this approach is that the vasculature is predominantly associated with the stroma of the breast and not in the adipose tissue. We used this 2-D area fraction as an estimate of the 3 -D volume fraction, which is most accurate when the ducts seen in the tissue block are predominantly cross sections of tubular structures. Because of difficulties in estimating $f$ from individual sections that may have included variable amounts of surrounding stroma, we averaged $f$ across all of the index series based on the assumption that duct density is relatively constant in the affected population.

Duct radius and viable-rim thickness were measured individually for each patient using a calibrated scale embedded in the pathology images (to take into account the effect of local cellular uptake on the oxygen concentration). Corresponding diffusion penetration lengths ranged in value from 77-224 $\mu \mathrm{m}$, which compare well with typical published values of $100 \mu \mathrm{m}$ under ideal conditions (e.g., [37]). The larger values corresponded to low grade cribriform DCIS where the local density of cells is less than it is for higher grade and more dense (solid) DCIS. This result corresponds to our expectations based on the relatively lower oxygen uptake by low-grade cribriform type DCIS (per unit tissue volume) due to the lower density of tumor cells. The lower density can be attributed to the type of DCIS, as cribriform type has internalized acinar-like spaces, and the lower PI values observed for low grade DCIS tumors.

These measurements for each case are given in Table S2 in the Supplementary Data. Because Eq. (4) assumes the presence of a peri-necrotic boundary, the model calibration described above is best suited to solid, cribriform and mixed solid-cribriform type DCIS, where a viable rim thickness can be readily identified, in contrast to micropapillary type DCIS, which is characterized by ducts with hollow centers. To be more consistent with the assumptions of Eq. (4), and thus to obtain more accurate parameter calibration, we excluded duct cross sections with non-necrotic hollow centers in the 2009 batch, whereas all samples were previously considered [30].

\section{Results}

Our simulation studies [1] of the growth of the DCIS surgical volumes over time (not shown) using input parameters calculated as in Methods indicate that more than $80 \%$ DCIS tumors, assuming that they are not palpable and therefore would not be self-diagnosed, should have reached at least $95 \%$ of their (quasi-)steady state surgical volumes by the time they were detected by yearly mammography screening, also assuming slow-to-non-varying tumor cell phenotypic properties during the course of their growth. This model prediction is consistent with published findings that nearly $80 \%$ of in-situ tumors identified by mammographic screening are either static with respect to size, or have very slow growth rates consistent with an approach to steady state [51, 52]. Thus we used Eq. (1) to compute the steady-state geometric-mean diameter of the surgical tumor volume from the parameters $A=\lambda_{\mathrm{A}} / \lambda_{\mathrm{M}}$ and $L$ according to the protocol described in Methods (Fig. 1) for comparison with tumors measurements from pathologic specimen studies and from the mammographic images. Note that for "large" tumors where $R$ exceeds the value of $L$, Eq. (1) reduces to a simple expression for the geometric mean diameter:

$$
2 R=6 \cdot L / A \text {. }
$$

Thus, for large tumors the surgical volume is directly proportional to the ratio of $L$ to $A$, where larger $L$ indicates adequate access to nutrients by diffusion deeper into the duct, and $A$ represents an index of cell death relative to cell proliferation (see Methods). Examining our measured values for $L$ and for $A$ (see Table 2), we can see that we are effectively in the range where this simplification applies. These values for $L$ represent the quantified effect that diffusion processes within tumor tissue have on the size each tumor can achieve.

As shown in Fig. 2 the simplified formula (Eq. 5) allows us to compare in physical units (i.e., centimeter) the tumor sizes (the "Path" column from Table 2) measured from pathologic specimens and plot these against $L / A$ values calculated from Ki-67 proliferative index, cleaved caspase-3 death index and viable rim thickness as per Eq. (2) (the error estimation is 
described in caption). Tumor sizes correlated with the parameter $L / A$ (linear correlation coefficient $\mathcal{R}^{2}=0.74$ by removing two outlier cases 8 and 14), as predicted by the simplified version (Eq. 5) of our model. Size increases proportional to the ratio $1 / A$ of cell mitosis over death and to the nutrient diffusion penetration length $L$, i.e., availability of nutrients limits tumor growth. This correlation demonstrates the predictive value of the biophysical model of tumor growth. A preliminary version of this figure was previously reported [30, Fig. 10.8], where the full formula (Eq. 1) was plotted against the 2008-batch of data only in a mathematically non-dimensionalized units.

Three cases (8, 14, 48 in Fig. 2) deviate significantly from the predictions of the model curve Eq. (5). In all three cases the estimated mean geometric diameter is greater than $5.01 \mathrm{~cm}$. In contrast, cases 8 and 14 are small tumors (geometric-mean diameter $\sim 1 \mathrm{~cm}$ ); closer examinations of the tissue samples of these two cases show relatively high proliferation and thick viable rims, suggesting substantial oxygenation within the tumors. Hence, they are likely still in the rapid-growth stage, where Eq. (1) (and therefore Eq. (5)) does not apply (see Methods). In fact, the com- puted final diameter for case 14 is nearly $70 \mathrm{~cm}$, which is physically impossible. For case 8 the computed geometric mean diameter is approximately $11 \mathrm{~cm}$, which would also be a very large tumor; for case 48 the geometric mean diameter is $5.8 \mathrm{~cm}$. Simulations of the time-dependent model [1] indicate that the tumor should reach nearly $90-95 \%$ of their final size stage by approximately 2 months for the values of $A$ measured in the 17 cases, thus predicting roughly a $2 / 12=17 \%$ probability of a tumor being diagnosed still in this stage using yearly mammogram screenings. This is consistent with the observation that these three cases represent $3 / 17$ or approximately $18 \%$ of cases examined here, thus corroborating the hypothesis that these three outliers should be indeed still in the early, more rapid growth phase. In addition, for these three cases the fewest tissue samples among the entire 17 cases were analyzed by IHC, which, together with the generally low frequency of apoptosis, potentially introduced a higher degree of significant error in the measurement of $A$.

Mammographic measurements demonstrate roughly equal numbers of overestimates versus underestimates of the tumor size, and are not a reliable

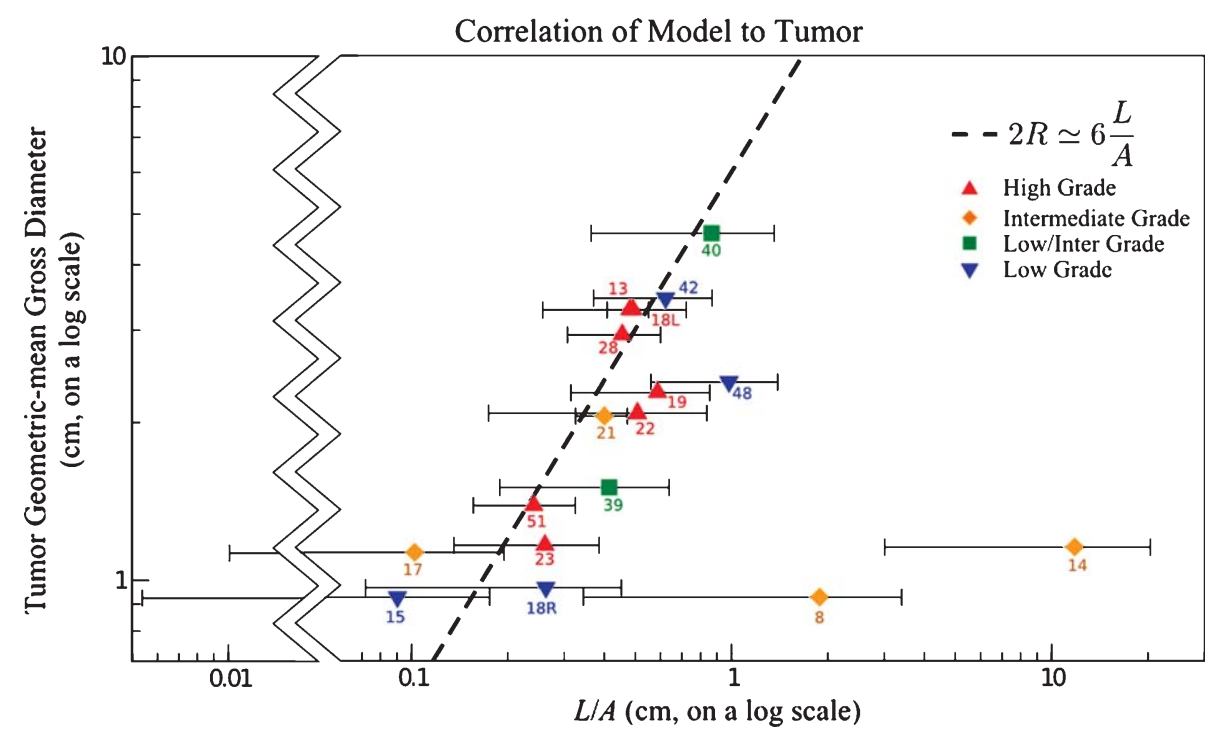

Fig. 2. Tumor size correlates with the death-to-proliferation ratio parameter. Tumor geometric-mean diameters $2 R$ (dashed) vs. $A / L$ predicted by Eq. (5) compared to the corresponding pathology measurements from the 17 excised tumors (symbols, with de-identified case numbers). Standard errors of the mean (SEMs) are reported [46, 47] (see Methods for details) (see also Table 2). SEMs for L/A were calculated as the summation of the first order in Taylor expansion from the variability of the AI and PI stains and the viable-rim thickness measurements in each tumor. For an explanation of cases 8, 14 and 48 see text of Results section. Previously [30, Fig. 10.8], we used the 2008 batch of patient tumor volume data alone (cases 8-28) to assess the feasibility and accuracy of a prototype parameter calibration protocol. 


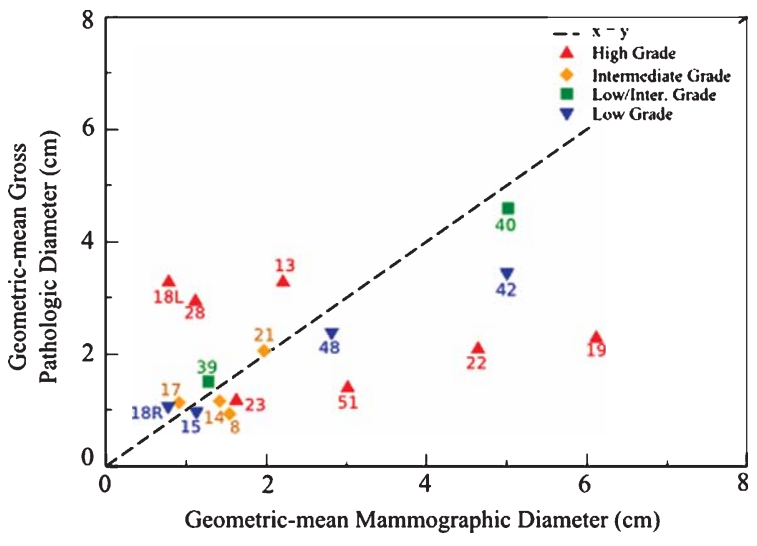

Fig. 3. Mammography, grade are poor predictors of tumor size. Comparison between the geometric-mean tumor dimension from mammograms and from pathology analysis of 17 excised DCIS tumors (de-identified case numbers are reported). Mammography overestimates tumor size in ten cases and underestimates the other seven. Correlations of both the mammography and the nuclear grade (in legend) with the tumor sizes are poor at best.

patient-specific predictor $\left(\mathcal{R}^{2}=0.47\right)$, as shown in Fig. 3. For example, specimen 19 has a geometric mean diameter of approximately $6 \mathrm{~cm}$ by mammography but only approximately $2 \mathrm{~cm}$ by pathological examination of the surgical specimen after excision. In contrast, specimen $18 \mathrm{~L}$ was less than $1 \mathrm{~cm}$ by mammography but was $>3 \mathrm{~cm}$ by pathologic analysis after excision (both are high grade tumors). See also Tables 2 and S3. Note that in both cases the size correlation between the pathologic examination and the model prediction are very close.

Histological grades also showed weak to no correlation with tumor sizes (Fig. $3 ; \mathcal{R}^{2}=0.08$ or the spearman rank correlation coefficient $\rho=0.16$ ). Consistent with previous work [53], the apoptotic and proliferative indices tracked similarly with grade of tumor (Fig. 4a; $\rho=0.82$ for PI and $\rho=0.77$ for AI). Note that these two indices show very similar trend when graphing index versus grade. Thus their ratio would be only weakly dependent on grade $(\rho=-0.26)$, corroborating the finding that net proliferation (i.e., ratio of PI to $\mathrm{AI}$ ) and thus tumor size should have a weaker correlation with grade.

Finally, viable-rim thickness of tumor in ducts and thus the nutrient diffusion penetration length $L$ decrease as a function of histological grade (Fig. 4b): more proliferative, high-grade tumors result in tightly packed patterns and thus are likely to hamper oxy-

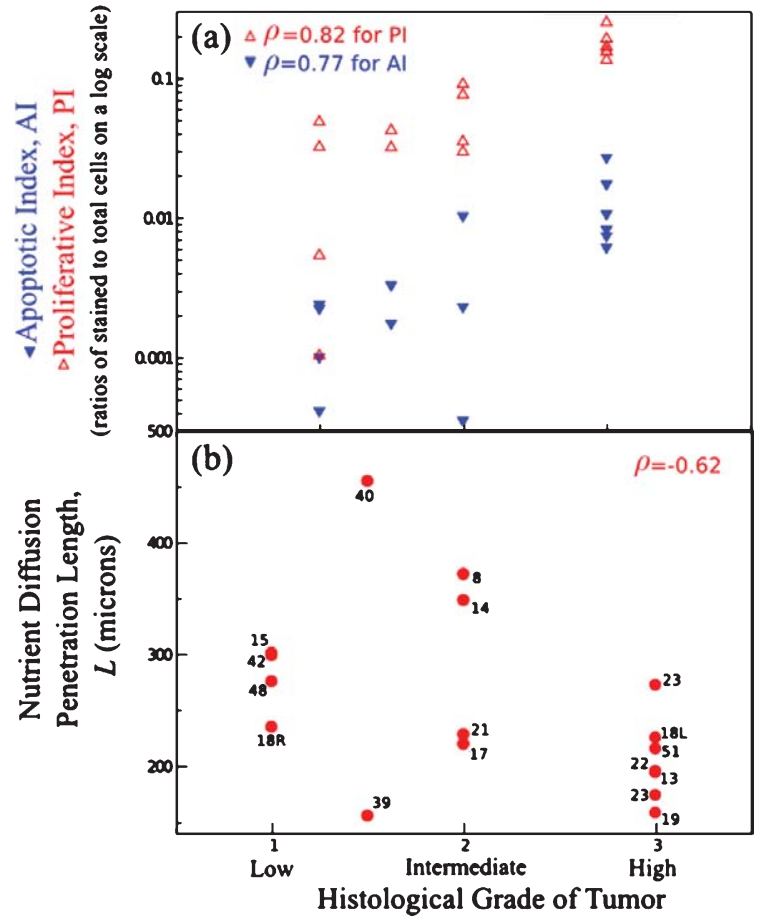

Fig. 4. Correlations of IHC and morphometric measurements with nuclear grade (with spearman rank correlation coefficients). (a) Average apoptotic (AI) and proliferative (PI) indices for each tumor as increasing functions of (modified Black's) nuclear grade (one overstained-AI tumor and one zero-AI tumor were excluded). (b) Nutrient diffusion penetration length $L$ from average measured viable-rim thickness $T$ in each tumor's ducts is a decreasing function of nuclear grade.

gen and nutrient diffusion. Diffusion limits tumor size. Lack of oxygen in these high-grade tumors would drive hypoxia-inducible factors and cell migration, leading to penetration of the ductal wall and infiltration of the tumor in the breast stroma, as has been previously reported [21, 22].

\section{Discussion}

The death-to-mitosis ratio $A$ is a strong predictor of tumor volume, thus suggesting that cell death immunohistochemical measurements should be performed on biopsied tissue (in addition to cell proliferation) to aid in the surgical planning. Studies seeking correlations between pathology based measurements of actual tumor volume and histological grade or tumor dimensions predicted from mammography indicate that these 
are unreliable sources for estimates of tumor size [54, 55]. Excluding the outlier cases 8 and 14, then the pathology based tumor sizes correlate with $L / A$ (linear correlation coefficient $\mathcal{R}^{2}=0.74$ ) as predicted by Eq. (5). In contrast the pathology based tumor size does not correlate with grade for the same cases $\left(\mathcal{R}^{2}=0.08\right)$. Considering that grade is a subjective assessment of nuclear size and nuclear pleomorphism and thus not a precise quantification, we also calculate the spearman rank correlation coefficient $\rho=0.16$ between tumor size and grade, still revealing a very weak correlation. Examining the data further, we find that grade does correlate with PI $(\rho=0.82)$ and $\mathrm{AI}(\rho=0.77)$. The correlation is less pronounced with $L(\rho=-0.62)$ and much weaker for $A(\rho=-0.26)$. Thus, while grade is informative with regard to PI it is not informative with respect to expected tumor size, while the ratio of $L / A$ is informative. If we only consider the cases where the mean geometric diameter is less than or equal to $5.01 \mathrm{~cm}$, then the correlation of $L / A$ with the geometric mean diameter of the pathology-based size is even better $\left(\mathcal{R}^{2}=0.88\right)$. In comparison, the correlation of the geometric mean diameter of the pathology-based size with the geometric mean diameter based on mammography measurements is poor $\left(\mathcal{R}^{2}=0.47\right.$ excluding the two outlier cases). It is the same when case 48 is also excluded.

Our findings demonstrate that morphology and other tumor characteristics (e.g., margin width, mammographic size, extent of involvement of the cores by DCIS, in addition to solid type, high grade, presence of necrosis, and presence of calcifications), which have been correlated with compromised surgical margins $[14,56]$, are histological surrogates for mechanistic parameters. Using the novel, biophysics-based approach applied here, we can more accurately predict surgical volumes in the form of explicit mathematical functions. We can conclude that the ratio of parameter $L$, the characteristic diffusion length from morphometric studies, to $A$, the death-to-mitosis ratio as determined by cleaved caspase- 3 and Ki- 67 staining, is a strong predictor of steady state tumor volume. In comparison, mammographic estimates have a poor correlation.

Our results also indicate that while high grade correlates with PI, it has poor correlation with $A, L$ and thus with tumor size. We note that we are able to obtain more accurate counts of both AI and PI from higher grade tumors, resulting in more accurate prediction of their steady-state tumor diameters (Fig. 2 and Table 2).
High-grade tumors are more likely to be associated with necrosis, which is required so that we can determine $L$ from the viable rim thickness. Thus, for high grade tumors diagnosed on core biopsy, measurements of $A$ and $L$ could not only be feasible, but they might be used to predict tumor volume with better accuracy than current methods. ${ }^{4}$

We can interpret these results as a biophysical explanation of how diffusion processes within the tumor tissue limit the maximum size that the tumor could achieve in an otherwise ideal environment with excess nutrients. Highly proliferative tumors with low apoptotic rates and with good penetration of nutrients will be larger; however, as the diffusion penetration length gets smaller then access to nutrients to support growth is negatively impacted and the expected tumor size decreases.

Despite the small number of tumor ducts used in this study to calculate the input parameters $A$ and $L$ to the biophysical model for each patient, the model predicted diameter and the pathology determined geometric mean diameter strongly correlate, demonstrating the potential of the mathematical pathology approach for translational application. In contrast, the image predicted geometric mean diameter has a poor correlation with the actual tumor geometric mean diameter determined from pathology. We envision future work with the model to validate a role in predicting surgical volumes required for adequate excision of DCIS, and for predicting whether there is a high likelihood of residual disease after excision with close margin based on the expected tumor volume versus the excised surgical volume.

One aspect of surgical planning that has not been addressed here is how shape impacts the individ-

\footnotetext{
${ }^{4}$ High grade when combined with presence of necrosis has been associated with an increased risk of invasion [57]. We note that the measured value for $L$ tends to cluster at a range of lower values for the high-grade tumors (see Fig. 3c and Table 2). High-grade tumors are more proliferative and result in ducts with more tightly packed cells. This higher cell density will hamper oxygen and nutrient diffusion to the inner part of the duct. When these tumors involve ducts with a radius larger than $L$, proliferation is reduced and ischemic necrosis occurs. Lack of oxygen has been postulated to drive hypoxia-inducible factors leading to penetration of the ductal wall and infiltration of the tumor in the breast stroma [21, 22]. This may explain the correlation of grade and necrosis with invasion. It does not explain the correlation of mammographic size with invasion, although this might be due to the fact that more extensive calcifications across the breadth of the tumor are an indicator of ongoing hypoxia and necrosis.
} 
ual dimensions once the surgical volume has been estimated. As can be seen from the individual mammographic measurements (see Table S3) these x-ray based images that detect calcifications in patterns associated with the Presence of DCIS are not very accurate in determining the actual size of a tumor, nor are they very accurate in predicting the relative axes of the tumors. It is likely that different size distributions of ducts are present along the three dimensions of the tumor, thus leading to different extents of necrosis along the different dimensions and therefore inaccurate relative dimensions. For example tumor in smaller ducts along one dimension may have sufficient oxygen to preclude ischemic necrosis. This dimension might be underestimated, while tumor in larger ducts growing in another dimension may be adequately measured because of necrosis. Calcifications within lesions such as columnar cell change confound the measurements. ${ }^{5}$ Other imaging modalities such as magnetic resonance imaging (MRI) or possibly ultrasound may be able to provide more accurate information regarding the relative proportions of the dimensions of a tumor. A model assisted 3dimensional image combining MRI and a predicted volume from the model may provide better technical assistance to the surgeon. Estimating the proximity of margins and the probability of tumor left in the patient in a 3-dimensional image may become increasingly important as the quantity of radiation is reduced in the adjuvant setting.

Breast architecture and the arborization of ducts, unique to each individual although on a common body plan, may also influence the actual geometry and thereby local extension of intraductal tumor cells. Imaging modalites other than mammography, such as MRI, ultrasound, and optical approaches, for the breast are rapidly coming generally available at affordable cost. Such newer imaging provides detailed information about duct architecture and could then be used as a map to input the simulated cancer growth derived from our model, thereby greatly enhancing the predictive power of the tumor size. Statistical approaches have also described a number of genes expressed in more aggressive DCIS [58] which could

\footnotetext{
${ }^{5}$ Mammography is not a reliable indicate because it is dependent upon calcifications and these can often be due to glandular secretions in some of the glands that have undergone columnar change. Only about $20 \%$ of biopsies have tumor present (invasive or in situ); hence most calcifications are benign.
}

be identified in biopsy samples and further empower our predictive model.

In addition to contributions to planning for treatment of high grade DCIS, the model also has implications in assessing the utility of screening mammography. The expected result of a screening program beginning at age 40 that would detect pre-invasive or early invasive cancers was a reduction in the number of invasive carcinomas or deaths from cancer later in life. A significant reduction has not been observed, leading public health officials to question the success of screening mammography. Specifically, a rapid growth phase (on a time scale of 2-3 months) is followed by a prolonged phase of slow growth (on a time scale of years) as tumor growth becomes hampered by diffusion gradients of cell nutrients. Using input parameters that represent a physiological range seen in DCIS tumors excised at the University of Texas MD Anderson Cancer Center, the model predicts that DCIS tumors will have reached a stationary volume within approximately three months [32]. Thus, within a year's time most of the in situ tumors will have reached their steady-state volume. If these tumors do not progress to an invasive state, it is unlikely that they will be detected by self-palpation prior to screening. In contrast, aggressive tumors that rapidly progress to an invasive state will begin a rapid growth phase again, now that their growth rate is no longer constrained by the need for nutrients to penetrate the intraductal space. We would expect these tumors to become large enough to be self-palpated prior to being detected on screening mammography. This expectation is reflected in the finding that symptom-detected breast cancers have more copy number imbalances, which is associated with more aggressive behavior [59]. Thus, the screening process is biased to detect less aggressive tumors that do not progress to an invasive state. Based on our estimates of the time to reach steady state, approximately $15 \%$ of tumors are detected prior to reaching steady state. The benefit to be realized in terms of reduced morbidity and mortality in subsequent decades would only be for the proportion of those tumors that were detected early $(15 \%)$ and that were likely to invade within one year $(67-75 \%$ based on SEER estimates of DCIS diagnosed with and without co-existing invasive carcinoma). This would result in a benefit of reduced morbidity and mortality in approximately $10 \%$ of the cases diagnosed early by screening. It may be that mammographic screen- 
ing, which is limited to yearly surveillance at least in part because of the risk incurred by radiation, may not be suitable as a screening tool for the timeframe over which DCIS may develop, reach steady state, and progress to invasive carcinoma. We are currently compiling these results for a forthcoming publication.

This study represents a proof of principle that it should be possible to incorporate a mathematical modeling step within current clinical practice to improve assessment of tumor volumes and thus the outcome of surgery. Since IHC and morphometric measurements are performed on patient-specific breast biopsies, the clinical value of this mathematical pathology approach is that the prediction and resulting surgical planning can be tailored for that particular patient.

\section{Acknowledgments}

MEE: NIH grant 2P30CA016672 for partial funding of the immunohistochemical stains and support from MDACC. ELB: NINDS NS046810 and NS062184, and NIGMS P50GM085. VC: Cullen Trust for Health Care; NIH-PSOC grants 1U54CA143907 and 1U54CA143837; NIH-ICBP grant 1U54CA149196; NSF grant DMS-0818104 UNM Spatio-Temporal Modeling Center for partial support. G. Tomaiuolo, J Kim, A Segura, S Sanga, ADM Broom, S Kaliki (UTHSC-H), and AKKL Kumar (MDACC), for designing the computerized image processing protocols [45] and for processing of the pathologic data. K-A Do (MDACC), M Royce and $\mathrm{H}$ Arias-Pulido (UNM CC) for useful discussions. A Thompson (University of Dundee, UK) and comments. R Bassett, Jr. (MDACC) for help with statistical evaluations. This research was approved by the Institutional Review Boards at UTHSC-H and MDACC.

\section{Supplementary data}

Tables S1 and S2 list the distribution (mean \pm standard deviation) of the raw immunohistochemistry and morphometric data for each of the 17 tumor cases. Table S3 lists the measured dimensions of the tumoraffected volumes of these 17 tumor cases.

The PI and AI of each duct were calculated as the ratios of the numbers of the positively stained $\mathrm{Ki}-67$ and Caspase- 3 cells to the total numbers of the cells within the duct cross sections on the examined slides.
We averaged these per-duct calculations over all the processed ducts for each case (Table S1: columns 2 and 3). Also reported are the numbers of the counted duct cross sections (Table S1: columns 4 for PI and 5 for AI), as well as the numbers of the total counted cells (Table S1: columns 6 for PI and 7 for AI). Cases 8-28 were counted using the grayscale algorithm, while cases 39-51 were processed by the k-mean algorithm. The algorithms occasionally fail to count certain duct cross sections, for which the cells were counted manually.

We calculated the average $R_{\text {duct }}$ and $T$ (Table $\mathrm{S} 2$ : columns 2 and 3 ) over only the ducts containing necrotic cores or entirely filled with viable tumors, excluding hollow ducts. Case 15 showed no necrotic cores, resulting in $\left\langle R_{\text {duct }}\right\rangle=\langle T\rangle$. Case 48 also resulted in $\left\langle R_{\text {duct }}\right\rangle=\langle T\rangle$ because its necrotic regions were relatively small. The number of ducts used for these measurements for each case is also reported (Table S2: column 4), where the sample sizes are mostly smaller than those of AI and PI because ducts with hollow centers are disqualified due to key assumptions of the model (see Methods).

The viable volume fractions $f$ (Table S2: column 5) were calculated as ratios of viable tumor volumes to the total DCIS volumes including the host tissues outside the ducts. We obtained $f$ using low-resolution $(40 \times)$ images that showed several ducts instead of highresolution $(100 \times)$ ones that focused on the vicinities of just one or two ducts that could consequently overestimate $f$. However, we still found that the calculation of $f$ was considerably affected by sampling the images. Given that our model is relatively less sensitive to $f$ (only through a square-root law in Eq. (2b)), we adopted the average $f$ over all the tumors for our calculation to increase robustness, which gave $\langle f\rangle=0.24$ with the current 17 cases.

We also calculated the tumor cell density in each duct by dividing the total tumor cell count by the viable rim area, and averaged these over all the processed ducts for each case (Table S2: column 6).

The volumes of the tumor-affected tissues for each case were estimated by pathologically examining the specimens of the excised tissue samples. The three dimensions (Table S3: columns 2-4) were respectively obtained along the AP (anterior-to-posterior), SI (superior-to-inferior), and TS (medial-lateral) axes relative to the breast. The mammographic estimations along the same three axes are also reported (Table S3: columns 5-7). 
Table S1

Raw immunohistochemistry data for each case

\begin{tabular}{|c|c|c|c|c|c|c|}
\hline \multirow[t]{2}{*}{$\begin{array}{l}\text { Case } \\
\text { ID }\end{array}$} & \multirow[t]{2}{*}{ PI (\%) } & \multirow[t]{2}{*}{$\mathrm{AI}(\%)$} & \multicolumn{2}{|c|}{$\begin{array}{l}\text { Number of } \\
\text { counted ducts }\end{array}$} & \multicolumn{2}{|c|}{$\begin{array}{l}\text { Number of total } \\
\text { cell counts }\end{array}$} \\
\hline & & & PI & AI & PI & AI \\
\hline 8 & $9.37 \pm 3.41$ & $0.24 \pm 0.23$ & 5 & 3 & 3178 & 3539 \\
\hline 13 & $25.90 \pm 5.80$ & $8.59 \pm 1.06^{*}$ & 3 & 5 & 2318 & 913 \\
\hline 14 & $7.87 \pm 3.43$ & $0.04 \pm 0.06$ & 8 & 10 & 11595 & 13335 \\
\hline 15 & $0.56 \pm 0.30$ & $0.10 \pm 0.12$ & 9 & 8 & 7311 & 4278 \\
\hline 17 & $3.08 \pm 1.97$ & $1.07 \pm 1.64$ & 11 & 7 & 5480 & 4778 \\
\hline 18.1 & $0.11 \pm 0.16$ & $0.04 \pm 0.07$ & 11 & 18 & 15308 & 15320 \\
\hline 18.2 & $13.99 \pm 1.95$ & $0.86 \pm 0.64$ & 4 & 6 & 2006 & 2753 \\
\hline 19 & $17.43 \pm 9.25$ & $0.64 \pm 0.42$ & 7 & 11 & 2398 & 3325 \\
\hline 21 & $3.64 \pm 1.82$ & 0 & 12 & 4 & 8514 & 4509 \\
\hline 22 & $16.08 \pm 7.06$ & $0.77 \pm 1.08$ & 13 & 10 & 6576 & 4581 \\
\hline 23 & $17.07 \pm 4.20$ & $2.81 \pm 3.01$ & 6 & 16 & 2631 & 5768 \\
\hline 28 & $19.78 \pm 4.64$ & $1.10 \pm 1.22$ & 33 & 28 & 9202 & 7881 \\
\hline 39 & $3.30 \pm 1.56$ & $0.18 \pm 0.16$ & 5 & 7 & 1891 & 4384 \\
\hline 40 & $4.39 \pm 4.39$ & $0.34 \pm 0.58$ & 24 & 31 & 22165 & 26588 \\
\hline 42 & $3.33 \pm 2.08$ & $0.25 \pm 0.24$ & 11 & 20 & 3429 & 12022 \\
\hline 48 & $5.03 \pm 1.27$ & $0.23 \pm 0.17$ & 6 & 5 & 3249 & 3040 \\
\hline 51 & $16.24 \pm 6.00$ & $1.81 \pm 1.59$ & 24 & 23 & 8256 & 11654 \\
\hline
\end{tabular}

* Over-stained samples.

Table S2

Raw morphometric data for each case

\begin{tabular}{llcccc}
\hline Case & $\begin{array}{c}\text { Duct radius } \\
R_{\text {duct }}(\mu \mathrm{m})\end{array}$ & $\begin{array}{c}\text { Viable rim } \\
\text { thickness } \\
\text { ID }\end{array}$ & $\begin{array}{c}T(\mu \mathrm{m}) \\
\text { Number of } \\
\text { measured } \\
\text { ducts } \\
f(\%)\end{array}$ & $\begin{array}{c}\text { Viable } \\
\text { volume } \\
\text { fraction }\end{array}$ & $\begin{array}{c}\text { Cell density } \\
\left(10^{5} \mathrm{cells}^{2} \mathrm{~cm}^{2}\right)\end{array}$ \\
\hline 8 & $422.58 \pm 51.00$ & $183.22 \pm 57.80$ & 3 & $36.59 \pm 2.81$ & $4.77 \pm 0.58$ \\
13 & $243.03 \pm 117.64$ & $96.43 \pm 31.95$ & 5 & $16.83 \pm 2.64$ & $2.79 \pm 0.54$ \\
14 & $204.53 \pm 45.28$ & $171.83 \pm 34.96$ & 8 & $23.89 \pm 8.41$ & $8.51 \pm 1.92$ \\
15 & $147.77 \pm 58.06$ & $147.77 \pm 58.06$ & 9 & $31.76 \pm 4.59$ & $8.83 \pm 1.58$ \\
17 & $115.86 \pm 41.80$ & $108.92 \pm 46.18$ & 11 & $19.60 \pm 12.94$ & $5.94 \pm 1.94$ \\
18.1 & $146.27 \pm 48.98$ & $116.35 \pm 42.40$ & 11 & $25.45 \pm 10.79$ & $11.20 \pm 1.84$ \\
18.2 & $232.75 \pm 55.86$ & $111.71 \pm 22.59$ & 4 & $29.46 \pm 7.67$ & $3.44 \pm 0.70$ \\
19 & $158.75 \pm 75.98$ & $78.87 \pm 12.53$ & 7 & $19.11 \pm 4.45$ & $3.21 \pm 0.60$ \\
21 & $120.68 \pm 73.45$ & $113.11 \pm 71.70$ & 12 & $21.12 \pm 5.50$ & $5.57 \pm 1.27$ \\
22 & $270.87 \pm 97.59$ & $97.08 \pm 25.85$ & 9 & $23.63 \pm 7.53$ & $4.52 \pm 1.13$ \\
23 & $157.62 \pm 73.13$ & $134.78 \pm 36.43$ & 6 & N/A & $4.03 \pm 0.36$ \\
28 & $135.51 \pm 81.34$ & $86.58 \pm 36.52$ & 33 & $26.85 \pm 9.06$ & $4.36 \pm 1.44$ \\
39 & $119.60 \pm$ N/A & $77.55 \pm$ N/A & 2 & $22.03 \pm 7.67$ & N/A** \\
40 & $323.17 \pm 33.22$ & $223.91 \pm 26.82$ & 3 & $24.07 \pm 6.08$ & N/A** \\
42 & $191.82 \pm$ N/A & $148.70 \pm$ N/A & 2 & $29.49 \pm 7.61$ & N/A** \\
48 & $136.28 \pm$ N/A & $136.28 \pm$ N/A & 1 & $7.61 \pm 4.95$ & N/A** \\
51 & $293.21 \pm 118.09$ & $106.91 \pm 24.24$ & 6 & $20.97 \pm 6.40$ & N/A** \\
\hline
\end{tabular}

** Cell density was not calculated for these cases.

Table S3

Measured dimensions for each case

\begin{tabular}{lccccccc}
\hline Case ID & \multicolumn{3}{c}{ Pathological dimensions $(\mathrm{cm})$} & & \multicolumn{3}{c}{ Mammographic dimensions $(\mathrm{cm})$} \\
\cline { 2 - 3 } & $\mathrm{AP}^{* * *}$ & $\mathrm{SI}^{* * *}$ & $\mathrm{TS}^{* * *}$ & & $\mathrm{AP}^{* * *}$ & $\mathrm{SI}^{* * *}$ & $\mathrm{TS}^{* * *}$ \\
\hline 8 & 0.9 & 1.1 & 0.8 & & 1.9 & 1.8 & 1.1 \\
13 & 4.0 & 3.5 & 2.5 & & 2.7 & 1.5 & 2.7 \\
\hline
\end{tabular}


Table S3

(Continued)

\begin{tabular}{|c|c|c|c|c|c|c|}
\hline \multirow[t]{2}{*}{ Case ID } & \multicolumn{3}{|c|}{ Pathological dimensions $(\mathrm{cm})$} & \multicolumn{3}{|c|}{ Mammographic dimensions $(\mathrm{cm})$} \\
\hline & $\mathrm{AP}^{* * *}$ & $\mathrm{SI}^{* * *}$ & $\mathrm{TS}^{* * *}$ & $\mathrm{AP}^{* * *}$ & $\mathrm{SI}^{* * *}$ & $\mathrm{TS}^{* * *}$ \\
\hline 14 & 1.2 & 0.8 & 1.6 & 2.0 & 2.1 & 0.7 \\
\hline 15 & 0.5 & 1.2 & 1.5 & 1.0 & 1.0 & 1.5 \\
\hline 17 & 0.5 & 1.1 & 2.6 & 1.0 & 1.0 & 0.8 \\
\hline 18.1 & 3.0 & 1.0 & 0.4 & 1.0 & 1.0 & 0.5 \\
\hline 18.2 & 5.0 & 2.8 & 2.5 & 0.8 & 0.8 & 0.8 \\
\hline 19 & 2.0 & 0.6 & 9.8 & 3.0 & 9.5 & 8.0 \\
\hline 21 & 0.9 & 2.4 & 4.0 & 2.0 & 1.5 & 2.6 \\
\hline 22 & 1.0 & 3.2 & 2.8 & 5.0 & 5.0 & 4.0 \\
\hline 23 & 0.5 & 2.1 & 1.5 & 2.1 & 1.0 & 2.1 \\
\hline 28 & 4.5 & 2.0 & 2.8 & 1.2 & 1.1 & 1.1 \\
\hline 39 & 0.5 & 4.5 & 1.5 & 1.5 & 0.8 & 1.8 \\
\hline 40 & 3.0 & 4.0 & 8.0 & 7.0 & 3.0 & 6.0 \\
\hline 42 & 2.0 & 3.0 & 6.8 & 5.0 & 5.0 & 5.0 \\
\hline 48 & 1.5 & 2.0 & 4.5 & 3.0 & 2.5 & 3.0 \\
\hline 51 & 1.1 & 1.1 & 2.2 & 3.2 & 3.6 & 2.4 \\
\hline
\end{tabular}

***AP: anterior-to-posterior, SI: superior-to-inferior, TS: transverse plane (medial-lateral).

\section{References}

[1] V. Cristini, J. Lowengrub and Q. Nie, Nonlinear simulation of tumor growth, J Math Biol 46 (2003), 191-224.

[2] H.B. Frieboes, X. Zheng, C.H. Sun, B. Tromberg, R. Gatenby and V. Cristini, An integrated computational/experimental model of tumor invasion, Cancer Research 66 (2006), 1597-1604.

[3] M.D. Szeto, G. Chakraborty, J. Hadley, R. Rockne, M. Muzi, E.C. Alvord Jr., Krohn, A.M. Spence and K.R. Swanson, Quantitative metrics of net proliferation and invasion link biological aggressiveness assessed by MRI with hypoxia assessed by FMISO-PET in newly diagnosed glioblastomas, Cancer Research 69 (2009), 4502-4509.

[4] C.H. Wang, J.K. Rockhill, M. Mrugala, D.L. Peacock, A. Lai, K. Jusenius, J.M. Wardlaw, T. Cloughesy, A.M. Spence, R. Rockne, E.C. Alvord Jr. and K.R. Swanson, Prognostic significance of growth kinetics in newly diagnosed glioblastomas revealed by combining serial imaging with a novel biomathematical model, Cancer Research 69 (2009), 9133-9140.

[5] H. Hatzikirou, A. Chauviere, J.S. Lowengrub, J.D. Groot and V. Cristini, The effect of vascularization on glioma growth, Tumor Vascularture: Molecular, cellular, and tissue level aspects and implications, in: Tumor Vasculature: Molecular, Cellular, and Tissue level Aspects and Implications T. Jackson, ed., Springer, New York (2011).

[6] W.D. Dupont and D.L. Page, Risk factors for breast cancer in women with proliferative breast disease, $N$ Engl J Med 312 (1985), 146-151.

[7] D.L. Page, W.D. Dupont, L.W. Rogers and M. Landenberger, Intraductal carcinoma of the breast: Follow-up after biopsy only, Cancer 49 (1982), 751-758.

[8] M.E. Sanders, P.A. Schuyler, W.D. Dupont and D.L. Page, The natural history of low-grade ductal carcinoma in situ of the breast in women treated by biopsy only revealed over 30 years of long-term follow-up, Cancer 103 (2005), 2481-2484.

[9] J.F. Simpson and D.L. Page, Pathology of preinvasive and excellent prognosis breast cancer, Curr Opin Oncol 13 (2001), 426-430.

[10] L.C. Collins, R.M. Tamimi, H.J. Baer, J.L. Connolly, G.A. Colditz and S.J. Schnitt, Outcome of patients with ductal carcinoma in situ untreated after diagnostic biopsy: Results from the Nurses' Health Study, Cancer 103 (2005), 1778-1784.

[11] V. Eusebi, E. Feudale, M.P. Foschini, A. Micheli, A. Conti, C. Riva, S. Di Palma and F. Rilke, Long-term follow-up of in situ carcinoma of the breast, Semin Diagn Pathol 11 (1994), 223-235.

[12] N. Cabioglu, K.K. Hunt, A.A. Sahin, H.M. Kuerer, G.V. Babiera, S.E. Singletary, G.J. Whitman, M.I. Ross, F.C. Ames, B.W. Feig, T.A. Buchholz and F. Meric-Bernstam, Role for intraoperative margin assessment in patients undergoing breast-conserving surgery, Ann Surg Oncol 14 (2007), 1458-1471.

[13] L. Cheng, N.K. Al-Kaisi, N.H. Gordon, A.Y. Liu, F. Gebrail and R.R. Shenk, Relationship between the size and margin status of ductal carcinoma in situ of the breast and residual disease, J Natl Cancer Inst 89 (1997), 1356-1360.

[14] M.F. Dillon, A.A. Maguire, E.W. McDermott, C. Myers, A.D. Hill, A. O'Doherty and C.M. Quinn, Needle core biopsy characteristics identify patients at risk of compromised margins in breast conservation surgery, Mod Pathol 21 (2008), 39-45.

[15] N. Patani, B. Cutuli and K. Mokbel, Current management of DCIS: A review, Breast Cancer Res Treat 111 (2008), 1-11.

[16] M.J. Silverstein, Predicting residual disease and local recurrence in patients with ductal carcinoma in situ, J Natl Cancer Inst 89 (1997), 1330-1331.

[17] E.M. Nichols, A.A. Dhople, M.M. Mohiuddin, T.W. Flannery, C.X. Yu and W.F. Regine, Comparative analysis of the post- 
lumpectomy target volume versus the use of pre-lumpectomy tumor volume for early-stage breast cancer: Implications for the future, Int J Radiation Oncology Biol Phys 77 (2010), 197-202.

[18] A. Bankhead, 3rd, N.S. Magnuson and R.B. Heckendorn, Cellular automaton simulation examining progenitor hierarchy structure effects on mammary ductal carcinoma in situ, J Theor Biol 246 (2007), 491-498.

[19] S.J. Franks, H.M. Byrne, H.S. Mudhar, J.C. Underwood and C.E. Lewis, Mathematical modelling of comedo ductal carcinoma in situ of the breast, Math Med Biol 20 (2003), 277-308.

[20] S.J. Franks, H.M. Byrne, J.C. Underwood and C.E. Lewis, Biological inferences from a mathematical model of comedo ductal carcinoma in situ of the breast, J Theor Biol 232 (2005), 523-543.

[21] R.A. Gatenby, E.T. Gawlinski, A.F. Gmitro, B. Kaylor and R.J. Gillies, Acid-mediated tumor invasion: A multidisciplinary study, Cancer Res 66 (2006), 5216-5223.

[22] R.A. Gatenby, K. Smallbone, P.K. Maini, F. Rose, J. Averill, R.B. Nagle, L. Worrall and R.J. Gillies, Cellular adaptations to hypoxia and acidosis during somatic evolution of breast cancer, Br J Cancer 97 (2007), 646-653.

[23] Y. Kim, M.A. Stolarska and H.G. Othmer, The role of the microenvironment in tumor growth and invasion, Prog Biophys Mol Biol 106 (2011), 353-379.

[24] P. Macklin, M.E. Edgerton, A.M. Thompson and V. Cristini, Patient-calibrated agent-based modelling of ductal carcinoma in situ (DCIS): From microscopic measurements to macroscopic predictions of clinical progression, J Theor Biol (in review).

[25] K.A. Norton, M. Wininger, G. Bhanot, S. Ganesan, N. Barnard and T. Shinbrot, A 2D mechanistic model of breast ductal carcinoma in situ (DCIS) morphology and progression, $J$ Theor Biol 263 (2010), 393-406.

[26] K.A. Rejniak and A.R. Anderson, A computational study of the development of epithelial acini: II. Necessary conditions for structure and lumen stability, Bull Math Biol 70 (2008), 1450-1479.

[27] K.A. Rejniak and A.R. Anderson, A computational study of the development of epithelial acini: I. Sufficient conditions for the formation of a hollow structure, Bull Math Biol 70 (2008), 677-712.

[28] A.S. Silva, R.A. Gatenby, R.J. Gillies and J.A. Yunes, A quantitative theoretical model for the development of malignancy in ductal carcinoma in situ, J Theor Biol 262 (2010), 601-613.

[29] L. Sontag and D.E. Axelrod, Evaluation of pathways for progression of heterogeneous breast tumors, J Theor Biol 232 (2005), 179-189.

[30] P. Macklin, M.E. Edgerton and V. Cristini, Agent-based cell modeling: Application to breast cancer, in: Multiscale Modeling of Cancer: An Integrated Experimental and Mathematical Modeling Approach, V. Cristini and J. Lowengrub, Cambridge University Press, Cambridge, (2010) pp. 206-234.

[31] H. Frieboes, M.E. Edgerton, J.P. Fruehauf, F.R.A.J. Rose, L.K. Worral, R. Gatenby, M. Ferrari and V. Cristini, Prediction of drug response in breast cancer using integrative experimental/computational modeling, Cancer Res 69 (2009), 4484-4492.
[32] M. Edgerton, Y. Chuang, P. Macklin, S. Sanga, J. Kim, G. Tomaiuolo, Y. Wang, A. Broom, K. Do and V. Cristini, Using mathematical models to understand the time dependence of the growth of ductal carcinoma in situ, Cancer Research 69 (Suppl 2) (2009), 1165.

[33] P. Macklin, J. Kim, G. Tomaiuolo, M.E. Edgerton and V. Cristini, Agent-based modeling of ductal carcinoma in situ: application to patient-specific breast cancer modeling, in: Computational Biology: Issues and Applications in Oncology, T. Pham, eds., Springer, New York, (2009), pp. 77-112.

[34] P. Macklin, M.E. Edgerton, J.S. Lowengrub and V. Cristini, Discrete cell modeling, in: Multiscale Modeling of Cancer: An Integrated Experimental and Mathematical Modeling Approach, V. Cristini and J. Lowengrub, Cambridge University Press, Cambridge, 2010, pp. 88-122.

[35] H. Kuerer, C. Albarracin, W. Yang, R. Cardiff, A. Brewster, W. Symmans, N. Hylton, L. Middleton, S. Krishnamurthy, G. Perkins, G. Babiera, M. Edgerton, B. Czerniecki, B. Arun and G. Hortobagyi, Ductal carcinoma in situ, state of the science and roadmap to advance the field, J Clin Onc 27 (2009), 279-288.

[36] K.D. Mannes, M.E. Edgerton, J.F. Simpson, R.A. Jensen and D.L. Page, pagetoid spread in ductal Carcinoma in situ, characterization and computer simulation, United States and Conadian Academy of Pathology (USCAP) Annual Meeting 2002.

[37] M.R. Owen, H.M. Byrne and C.E. Lewis, Mathematical modelling of the use of macrophages as vehicles for drug delivery to hypoxic tumour sites, J Theor Biol 226 (2004), 377 391.

[38] S. Lee, S.K. Mohsin, S. Mao, S.G. Hilsenbeck, D. Medina and D.C. Allred, Hormones, receptors, and growth in hyperplastic enlarged lobular units: Early potential precursors of breast cancer, Breast Cancer Res 8 (2006), R6.

[39] J.L. Scarlett, P.W. Sheard, G. Hughes, E.C. Ledgerwood, H.H. $\mathrm{Ku}$ and M.P. Murphy, Changes in mitochondrial membrane potential during staurosporine-induced apoptosis in Jurkat cells, FEBS Lett 475 (2000), 267-272.

[40] Z. Hu, K. Yuri, H. Ozawa, H. Lu and M. Kawata, The in vivo time course for elimination of adrenalectomy-induced apoptotic profiles from the granule cell layer of the rat hippocampus, J Neurosci 17 (1997), 3981-3989.

[41] J.F. Kerr, C.M. Winterford and B.V. Harmon, Apoptosis. Its significance in cancer and cancer therapy, Cancer 73 (1994), 2013-2026.

[42] J.P. Ward and J.R. King, Mathematical modelling of avascular-tumour growth, IMA J Math Appl Med Biol 14 (1997), 39-69.

[43] J. Gerdes, H. Lemke, H. Baisch, H.H. Wacker, U. Schwab and H. Stein, Cell cycle analysis of a cell proliferation-associated human nuclear antigen defined by the monoclonal antibody Ki-67, J Immunology 133 (1984), 1710-1715.

[44] W.R. Duan, D.S. Garner, S.D. Williams, C.L. Funckes-Shippy, I.S. Spath and E.A.G. Blomme, Comparison of immunohistochemistry for activated caspase- 3 and cleaved cytokeratin 18 with the TUNEL method for quantification of apoptosis in histological sections of PC-3 subcutaneous xenografts, J Pathol 199 (2003). 
[45] Y.-1. Chuang, M.E. Edgerton, P. Macklin, J. Kim, A. Segura, G. Tomaiuolo, S. Sanga and V. Cristini, Bridging the gap between mathematical modeling and pathological data: An application of multiscale modeling to ductal carminoma in situ, in: Biophysical Modeling of Cancer in Silico V. Cristini, Springer, New York, 2012 (in preparation).

[46] G. Cumming, F. Fidler and D.L. Vaux, Error bars in experimental biology, J Cell Biol 177 (2007), 7-11.

[47] J.P. Morton, Reviewing scientific manuscripts: How much statistical knowledge should a reviewer really know? Adv Physiol Educ 33 (2009), 7-9.

[48] J.H. Menell, E.A. Morris, D.D. Dershaw, A.F. Abramson, E. Brogi and L. Liberman, Determination of the presence and extent of pure ductal carcinoma in situ by mammography and magnetic resonance imaging, Breast J 11 (2005), 382390.

[49] A. Grin, G. Horne, M. Ennis and F.P. O’Malley, Measuring extent of ductal carcinoma in situ in breast excision specimens: A comparison of 4 methods, Arch Pathol Lab Med 133 (2009), 31-37.

[50] L.P. Middleton, G. Vlastos, N.Q. Mirza, S. Eva and A.A. Sahin, Multicentric mammary carcinoma: Evidence of monoclonal proliferation, Cancer 94 (2002), 1910-1916.

[51] J. Spratt, L. Heuser, J.G. Kuhns, H.M. Reiman, J.B. Buchanan, H.C. Polk Jr. and J. Sandoz, Association between the actual doubling times of primary breast cancer with histopathologic characteristics and Wolfe's parenchymal mammographic patterns, Cancer 47 (1981), 2265-2268.

[52] J.Z. Thomson, A.J. Evans, S.E. Pinder, H.C. Burrell, A.R.M Wilson, and I.O. Ellis, Growth pattern of ductal carcinoma in situ (DCIS): A retrospective analysis based on mammographic findings, Br J Cancer 85 (2001), 225-227.
[53] A. Gandhl, P.A. Holland, W.F. Knox, C.S. Potten and N.J. Bundred, Evidence of significant apoptosis in poorly differentiated ductal carcinoma in situ of the breast, Br J Cancer 78 (1998), 788-794.

[54] S. Chan, J.H. Chen, G. Agrawal, M. Lin, R.S. Mehta, P.M. Carpenter, O. Nalcloglu and M.Y. Su, Characterization of pure ductal carcinoma in situ on dynamic contrast-enhanced MR Imaging: Do nonhigh grade and high grade show different imaging features?, J Oncol 2010 (2010), 431341.

[55] H. Liu and W. Peng, MRI morphological classification of ductal carcinoma in situ (DCIS) correlating with different biological behavior, Eur J Radiol (2011, in press).

[56] J. Chakrabarti, A.J. Evans, J. James, I.O. Ellis, S.E. Pinder and R.D. Macmillan, Accuracy of mammography in predicting histological extent of ductal carcinoma in situ (DCIS), Eur J Surg Oncol 32 (2006), 1089-1092.

[57] T.W. Yen, K.K. Hunt, M.I. Ross, N.Q. Mirza, G.V. Babiera, F. Meric-Bernstam, S.E. Singletary, W.F. Symmans, S.H. Giordano, B.W. Feig, F.C. Ames and H.M. Kuerer, Predictors of invasive breast cancer in patients with an initial diagnosis of ductal carcinoma in situ: A guide to selective use of sentinel lymph node biopsy in management of ductal carcinoma in situ, J Am Coll Surg 200 (2005), 516-526.

[58] E.S. Hwang, A. Lai, Y.-Y. Chen, S. DeVries, R. Swain, J. Anderson, R. Roy and F.M. Waldman, Genomic alterations and phenotype of large compared to small high-grade ductal carcinoma in situ, Hum Pathology (2011, in press).

[59] A.M. Brewster, P. Thompson, A.A. Sahin, K. Do, M.E. Edgerton, S. Tsavaschidis, R. Zhou, Y. Liu, L. Zhang, G. Mills and $\mathrm{M}$. Bondy, Copy number imbalances between screen and symptom-detected breast cancers and impact on disease-free survival, Cancer Prevention Research (2011, in press). 


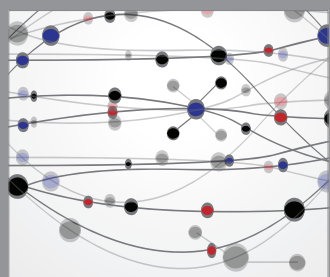

The Scientific World Journal
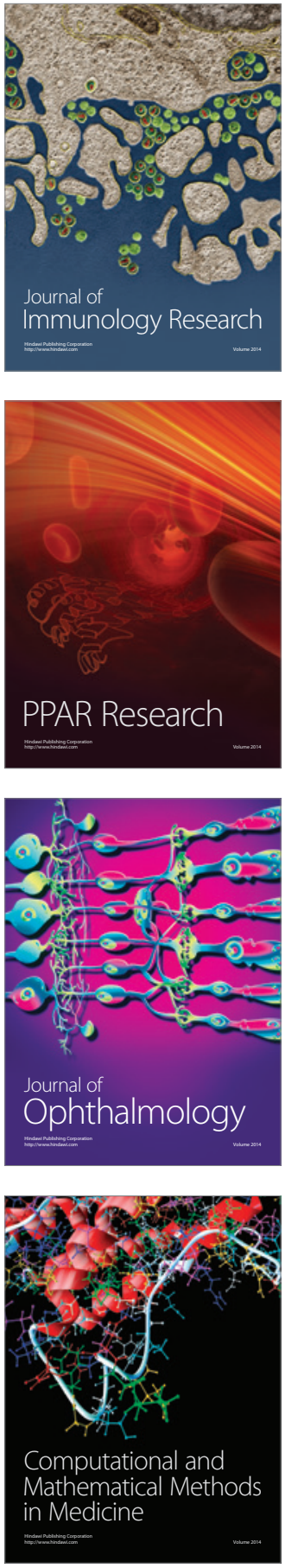

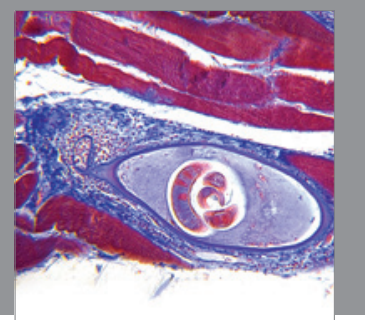

Gastroenterology

Research and Practice
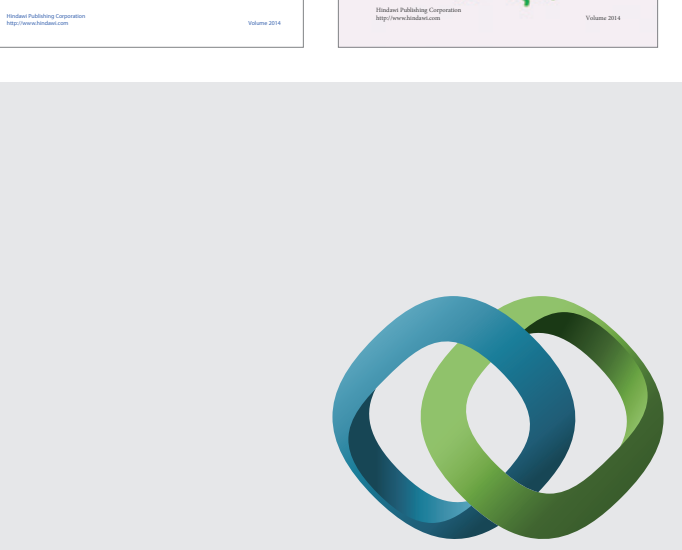

\section{Hindawi}

Submit your manuscripts at

http://www.hindawi.com
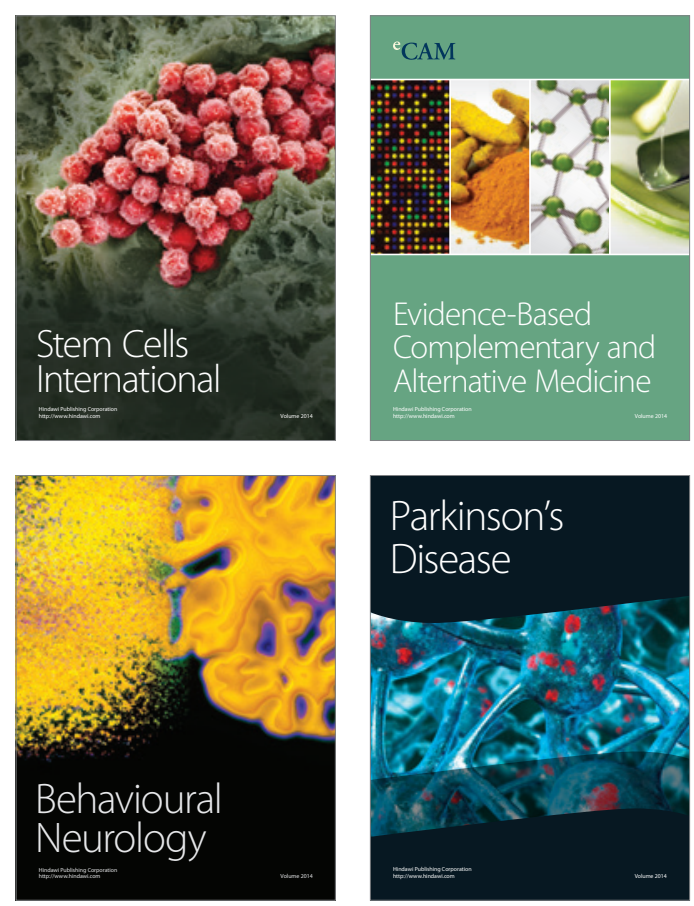

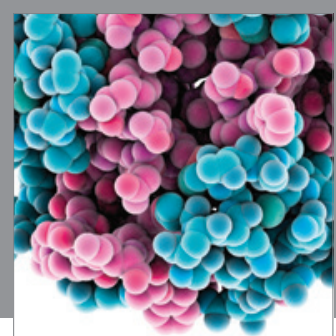

Journal of
Diabetes Research

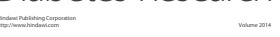

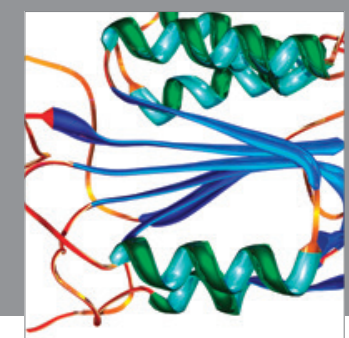

Disease Markers
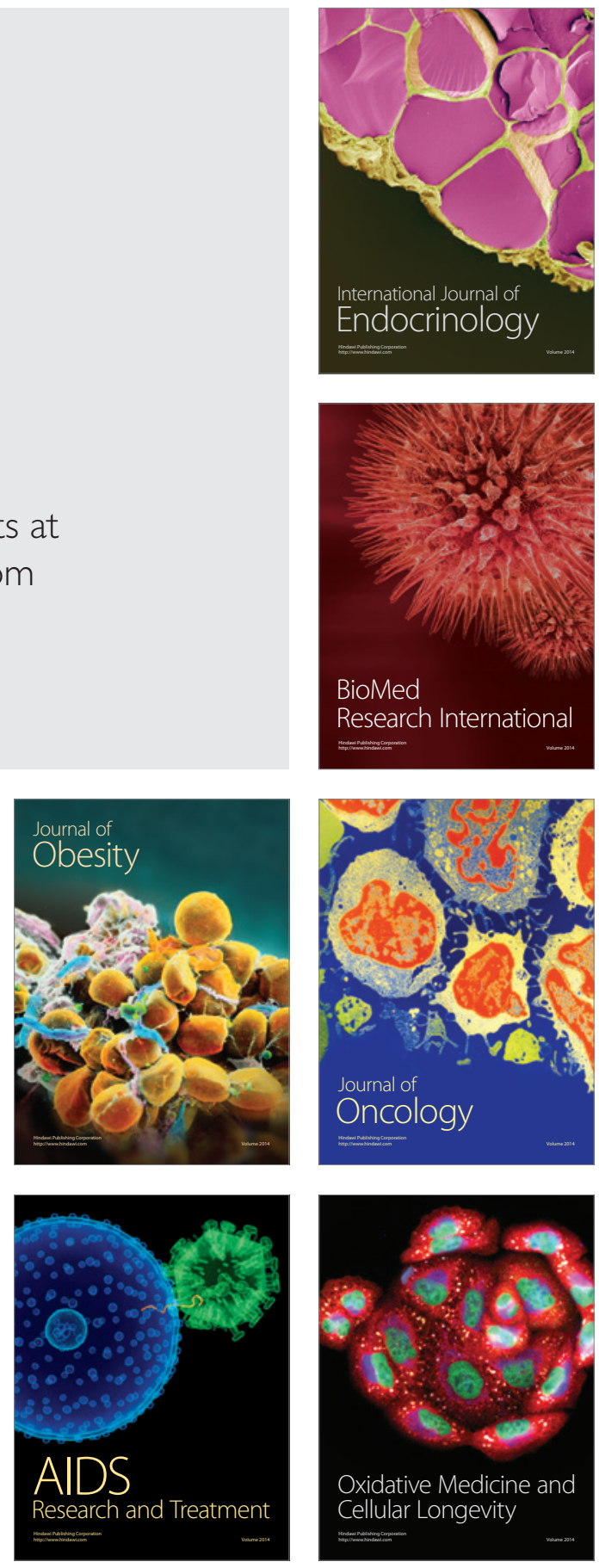